\title{
Orthogonal Block Structure and Uniformly Best Linear Unbiased Estimators
}

\author{
Sandra S. Ferreira, Dário Ferreira, Célia Nunes, Francisco Carvalho, \\ and João Tiago Mexia
}

\begin{abstract}
Models with orthogonal block structure, OBS, have variance covariance matrices that are linear combinations $\sum_{j=1}^{m} \gamma_{j} Q_{j}$ of known pairwise orthogonalorthogonal projection matrices that add up to $\boldsymbol{I}_{n}$. We are interested in characterizing such models with least square estimators that are best linear unbiased estimator whatever the variance components, assuming that $\gamma \in \nabla_{\geq}$, with $\nabla_{\geq}$the set of vectors with nonnegative components of a subspace $\nabla$. This is an extension of the usual concept of OBS in which we require $\boldsymbol{\gamma} \in \mathbb{R}_{\geq}^{m}$. Thus as we shall see it is usual when we apply our results to mixed models.
\end{abstract}

Keywords Best linear unbiased estimator - Least square estimators · Orthogonal block structure · Uniformly minimum variance unbiased estimator

\section{Introduction}

If a model has the family

$$
v=\left\{\sum_{i=1}^{w} \theta_{i} \boldsymbol{M}_{i} ; \quad \boldsymbol{\theta} \in \Theta\right\}
$$

\footnotetext{
S. S. Ferreira $(\varangle) \cdot$ D. Ferreira $\cdot$ C. Nunes

Department of Mathematics and Center of Mathematics and Applications, University of Beira Interior, Covilhã, Portugal

e-mail: sandraf@ubi.pt

F. Carvalho

Departmental Unit of Mathematics and Physics, Polytechnic Institute of Tomar and Center of Mathematics and Applications, FCT/UNL, Monte da Caparica, Portugal e-mail: fpcarvalho@ipt.pt

\section{J. T. Mexia}

Department of Mathematics and Center of Mathematics and Applications, FCT/UNL, Monte da Caparica, Portugal 
of variance-covariance matrices it suffices that $\boldsymbol{T}$, the projector onto the column space of matrix $\boldsymbol{X}_{0}$, commutes with $\boldsymbol{M}_{1}, \ldots, \boldsymbol{M}_{w}$ for the least square estimators, LSE, to be best linear unbiased estimator, BLUE, whatever $\boldsymbol{\theta}=\theta_{1}, \ldots, \theta_{w}$. Following [12] and [11], we say that, then, the LSE are uniformly best linear unbiased estimator, UBLUE.

When the model has only one variance component $\theta$, then having variancecovariance matrix $\theta \boldsymbol{M}, \boldsymbol{T}$ commuting with $\boldsymbol{M}$ is, see [13] and [14], a necessary and sufficient condition for the LSE to be UBLUE. Then matrix $\boldsymbol{M}$ has the spectral decomposition

$$
\boldsymbol{M}=\sum_{j=1}^{m} b_{j} \boldsymbol{Q}_{j}
$$

with $\boldsymbol{Q}_{1}, \ldots, \boldsymbol{Q}_{m}$ pairwise orthogonal-orthogonal projection matrices, POOPM, and the family of variance-covariance matrices can be written as

$$
v=\left\{\sum_{j=1}^{m} \gamma_{j} \boldsymbol{Q}_{j} ; \quad \boldsymbol{\gamma} \in R(\boldsymbol{b})_{\geq}\right\}
$$

where $R(\boldsymbol{U})$ is the range space of matrix $\boldsymbol{U}, \boldsymbol{b}[\boldsymbol{\gamma}]$ has components $b_{1}, \ldots, b_{m}$ $\left[\gamma_{1}, \ldots, \gamma_{m}\right]$ and $\Delta_{\geq}$is the family of vectors of subspace $\Delta$ with nonnegative components. We say that those models have $\operatorname{rank} 1$, since $\operatorname{rank}(\boldsymbol{b})=1$. We intend to extend the necessary and sufficient conditions obtained for rank 1 models to models with

$$
v=\left\{\sum_{j=1}^{m} \gamma_{j} \boldsymbol{Q}_{j} ; \quad \boldsymbol{\gamma} \in \nabla \geq\right\},
$$

where $\operatorname{dim}(\nabla)=r \geq 1$. These models will have rank $r$. When $\nabla=\mathbb{R}^{m}$ all the matrices $\sum_{j=1}^{m} \gamma_{j} \boldsymbol{Q}_{j}$ with nonnegative coefficients may be variance-covariance matrices and we say the model is full rank. Moreover if

$$
\sum_{j=1}^{m} \boldsymbol{Q}_{j}=\boldsymbol{I}_{n}
$$

the model will have orthogonal block structure, OBS, see [8, 9]. These models continue to play a prominent role in the theory of randomized block designs, see $[2,3]$.

An interesting case studied by [1] is the case where the family of possible variance-covariance matrices, while still commutative, no longer forms an orthogonal block structure. 
In the next section we present results on commutative Jordan algebras (of symmetric matrices), CJA, and describe the algebraic structure of the model. Finally, in the third section, we characterize the models with OBS whose LSE are UBLUE, this is they are BLUE whatever the variance components.

\section{Algebras and Structure}

A CJA is a linear space constituted by symmetric matrices that commute and containing the squares of its matrices. Each one of these algebras, say $\mathcal{A}$, has a unique basis, the principal basis, $p b(\mathcal{A})$, constituted by POOPM, see [10]. For a family $\boldsymbol{W}=\left\{\boldsymbol{W}_{1}, \ldots, \boldsymbol{W}_{u}\right\}$ of symmetric matrices to be contained in a CJA, see, e.g., [5], it is necessary and sufficient that its matrices commute. Moreover, intersecting all the CJA that contain $\boldsymbol{W}$ we obtain the least CJA, $\mathcal{A}(\boldsymbol{W})$, that contains $\boldsymbol{W}$, this will be the CJA generated by $\boldsymbol{W}$. If the $n \times n$ matrices in $p b(\mathcal{A})$ add up to $\boldsymbol{I}_{n}$, the CJA will be complete. For a CJA to contain invertible matrices it is necessary and sufficient that it is complete, see [5].

Let us consider the mixed model

$$
\boldsymbol{Y}=\sum_{i=0}^{w} \boldsymbol{X}_{i} \boldsymbol{\beta}_{i}
$$

where $\boldsymbol{\beta}_{0}$ is fixed and the $\boldsymbol{\beta}_{1}, \ldots, \boldsymbol{\beta}_{w}$ are random, independent, with null mean vectors and variance-covariance matrices $\boldsymbol{\theta}_{1} \boldsymbol{I}_{c_{1}}, \ldots, \boldsymbol{\theta}_{w} \boldsymbol{I}_{c_{w}}$. If the matrices $\boldsymbol{M}_{i}=$ $\boldsymbol{X}_{i} \boldsymbol{X}_{i}^{\top}, i=1, \ldots, w$ commute, they will generate $\mathcal{A}=\mathcal{A}(\underline{\boldsymbol{M}})$, where $\underline{\boldsymbol{M}}=$ $\left\{\boldsymbol{M}_{1}, \ldots, \boldsymbol{M}_{w}\right\}$. With $\underline{\boldsymbol{Q}}=\left\{\boldsymbol{Q}_{1}, \ldots, \boldsymbol{Q}_{m}\right\}=p b(\mathcal{A})$, we will have

$$
\boldsymbol{M}_{i}=\sum_{j=1}^{m} b_{i, j} \boldsymbol{Q}_{j}, i=1, \ldots, w
$$

and so we will have the variance-covariance matrices

$$
\boldsymbol{V}(\boldsymbol{\theta})=\sum_{i=1}^{w} \theta_{i} \boldsymbol{M}_{i}=\sum_{j=1}^{m}\left(\sum_{i=1}^{w} b_{i, j} \theta_{i}\right) \boldsymbol{Q}_{j}=\sum_{j=1}^{m} \gamma_{j} \boldsymbol{Q}_{j}=\boldsymbol{V}(\boldsymbol{\gamma}),
$$

with

$$
\boldsymbol{\gamma}_{j}=\sum_{i=1}^{w} b_{i, j} \theta_{i}, j=1, \ldots, m
$$

and so $\boldsymbol{\gamma} \in R\left(\boldsymbol{B}^{\top}\right)_{\geq}$, where $\boldsymbol{B}=\left[b_{i, j}\right]$. 
Let us establish

Proposition 1 If $R\left(\left[\boldsymbol{X}_{1} \ldots \boldsymbol{X}_{w}\right]\right)=\mathbb{R}^{n}$ and the matrices $\boldsymbol{M}_{i}, i=1, \ldots, w$, commute, the model has $O B S$.

Proof Since the $\boldsymbol{Q}_{1}, \ldots, \boldsymbol{Q}_{m}$ are POOPM we have only to show that $\sum_{j=1}^{m} \boldsymbol{Q}_{j}=$ $\boldsymbol{I}_{n}$, this is that $\mathcal{A}$ is complete. Now

$$
\operatorname{rank}\left(\sum_{i=1}^{w} \boldsymbol{M}_{i}\right)=\operatorname{rank}\left(R\left[\boldsymbol{X}_{1} \ldots \boldsymbol{X}_{w}\right]\right)=n,
$$

so $\sum_{i=1}^{w} \boldsymbol{M}_{i}$, being an $n \times n$ matrix with rank $n$, is invertible and since $\sum_{i=1}^{w} \boldsymbol{M}_{i} \in$ $\mathcal{A}, \mathcal{A}$ is complete.

We point out that $\boldsymbol{V}\left(\boldsymbol{\theta}_{1}\right)=\boldsymbol{V}\left(\boldsymbol{\theta}_{2}\right)$ implies $\boldsymbol{\theta}_{1}=\boldsymbol{\theta}_{2}$ if and only if the matrices $\boldsymbol{M}_{1}, \ldots, \boldsymbol{M}_{w}$ are linearly independent so the row vectors of matrix $\boldsymbol{B}$. From now on we make this assumption of linear independence so $\boldsymbol{B}$ will be a $w \times m$ matrix with rank $w$.

If the model has OBS and $\boldsymbol{T}$ commutes with $\boldsymbol{M}$, the model will have commutative OBS and we say that it has COBS. The models with COBS were introduced in [6]. We now have the

Proposition 2 A model with OBS has COBS if and only if $\boldsymbol{T}$ commutes with the $\boldsymbol{Q}_{1}, \ldots, \boldsymbol{Q}_{m}$.

Proof We have only to establish the part of the thesis for COBS since the proof for OBS is identical. For this, it is sufficient to show that $\boldsymbol{T}$ commutes with $\boldsymbol{M}_{1}, \ldots, \boldsymbol{M}_{w}$ if and only if it commutes with $\boldsymbol{Q}_{1}, \ldots, \boldsymbol{Q}_{m}$. Now, if $\boldsymbol{T}$ and the $\boldsymbol{M}_{1}, \ldots, \boldsymbol{M}_{w}$ commute, the matrices of $\boldsymbol{M}^{*}=\left\{\boldsymbol{T}, \boldsymbol{M}_{1}, \ldots, \boldsymbol{M}_{w}\right\}$ generate a CJA, $\mathcal{A}^{*}$, that contains $\mathcal{A}(\underline{\boldsymbol{M}})$, since $\underline{\boldsymbol{M}} \subseteq \underline{\boldsymbol{M}^{*}}$. Namely we will have $\boldsymbol{T}, \boldsymbol{Q}_{1}, \ldots, \boldsymbol{Q}_{m} \in$ $\mathcal{A}^{*}$ so $\boldsymbol{T} \boldsymbol{Q}_{j}=\widehat{\boldsymbol{Q}_{j} \boldsymbol{T}}, j=\overline{1, \ldots, m}$. The inverse is easy to establish since $\boldsymbol{M}_{i}=\sum_{j=1}^{m} b_{i, j} \boldsymbol{Q}_{j}, i=1, \ldots, w$, thus $\boldsymbol{T} \boldsymbol{Q}_{j}=\boldsymbol{Q}_{j} \boldsymbol{T}, j=1, \ldots, m$ implies $\boldsymbol{T} \boldsymbol{M}_{i}=\boldsymbol{M}_{i} \boldsymbol{T}, i=1, \ldots, w$.

Corollary 1 A model with OBS has COBS if and only if their matrices $Q_{j}^{*}=$ $\boldsymbol{Q}_{j} \boldsymbol{T}, j=1, \ldots, m$, are orthogonal projection matrices (we point out that $\mathbf{0}_{n \times n}$ is an orthogonal projection matrix).

Proof The thesis follows directly from Proposition 2 since the $\boldsymbol{Q}_{j}^{*}$ are symmetric and idempotent if and only if $\boldsymbol{Q}_{j} \boldsymbol{T}=\boldsymbol{T} \boldsymbol{Q}_{j}, j=1, \ldots, m$. We point out that, see [7], $p b\left(\mathcal{A}^{*}\right)$ is constituted by the nonnull matrices $\boldsymbol{T} \boldsymbol{Q}_{j}$ and $\left(\boldsymbol{I}_{n}-\boldsymbol{T}\right) \boldsymbol{Q}_{j}, j=$ $1, \ldots, m$.

Let the $g_{j}$ row vectors of matrix $\boldsymbol{A}_{j}$ constitute an orthonormal basis for $\nabla_{j}=$ $R\left(\boldsymbol{Q}_{j}\right)$. Now

$$
\widetilde{\psi}=\boldsymbol{U} \boldsymbol{Y}
$$


is an LSE estimator of its mean vector

$$
\psi=\boldsymbol{U} \boldsymbol{\mu}
$$

if and only if

$$
\boldsymbol{U} \boldsymbol{T}=\boldsymbol{U}
$$

We now have

Theorem 1 The OBS whose LSE are UBLUE are the COBS.

Proof As for Proposition 2 we have only to establish the first part of the thesis. In COBS we have, whatever $\boldsymbol{\gamma}, \quad \boldsymbol{T} \boldsymbol{V}(\boldsymbol{\gamma})=\boldsymbol{V}(\boldsymbol{\gamma}) \boldsymbol{T}$ as well as $\boldsymbol{T}^{c} \boldsymbol{V}(\boldsymbol{\gamma})=\boldsymbol{V}(\boldsymbol{\gamma}) \boldsymbol{T}^{c}$, with $\boldsymbol{T}^{c}=\boldsymbol{I}_{n}-\boldsymbol{T}$. Putting $\boldsymbol{U}_{\Omega}=\boldsymbol{U} \boldsymbol{T}$ and $\boldsymbol{U}_{\Omega^{\perp}}=\boldsymbol{U} \boldsymbol{T}^{c}$ we get

$$
\begin{gathered}
\operatorname{Cov}(\boldsymbol{U} \boldsymbol{Y})=\boldsymbol{U} \boldsymbol{V}(\boldsymbol{\gamma}) \boldsymbol{U}^{\top}=\left(\boldsymbol{U}_{\Omega} \boldsymbol{U}_{\Omega^{\perp}}\right) \boldsymbol{V}(\boldsymbol{\gamma})\left(\boldsymbol{U}_{\Omega^{\top}}^{\top} \boldsymbol{U}_{\Omega^{\perp}}^{\top}\right)= \\
=\boldsymbol{U}_{\Omega} \boldsymbol{V}(\boldsymbol{\gamma}) \boldsymbol{U}_{\Omega^{\perp}}^{\top} \boldsymbol{U}_{\Omega} \boldsymbol{V}(\boldsymbol{\gamma}) \boldsymbol{U}_{\Omega^{\perp}}^{\top},
\end{gathered}
$$

since $\boldsymbol{U}_{\Omega} \boldsymbol{V}(\boldsymbol{\gamma}) \boldsymbol{U}_{\Omega^{\perp}}^{\top}=\boldsymbol{U} \boldsymbol{T} \boldsymbol{V}(\boldsymbol{\gamma}) \boldsymbol{T}^{c} \boldsymbol{U}^{\top}=\boldsymbol{U} \boldsymbol{V}(\boldsymbol{\gamma}) \boldsymbol{T} \boldsymbol{T}^{c} \boldsymbol{U}^{\top}=\mathbf{0}_{n \times n}$ and, likewise $\boldsymbol{U}_{\Omega^{\perp}} \boldsymbol{V}(\boldsymbol{\gamma}) \boldsymbol{U}_{\Omega}^{\top}=\mathbf{0}_{n \times n}$, considering $\operatorname{Cov}$ the covariance matrix.

Given another linear unbiased estimator $\boldsymbol{\psi}^{*}=\boldsymbol{L} \boldsymbol{Y}$ of $\boldsymbol{\psi}$ we have $\boldsymbol{L} \boldsymbol{\mu}=\boldsymbol{U} \boldsymbol{\mu}$, so $\left(\boldsymbol{L}_{\Omega}-\boldsymbol{U}_{\Omega}\right) \boldsymbol{X}_{0}=(\boldsymbol{L}-\boldsymbol{U}) \boldsymbol{T} \boldsymbol{X}_{0}=\mathbf{0}_{n \times k}$ since the row vectors of $(\boldsymbol{L}-\boldsymbol{U}) \boldsymbol{T}$ belong to $\Omega=R\left(\boldsymbol{X}_{0}\right)$ and are orthogonal to $\Omega$.

Thus $\boldsymbol{L}_{\Omega}=\boldsymbol{L} \boldsymbol{T}=\boldsymbol{U} \boldsymbol{T}=\boldsymbol{U}_{\Omega}$, so

$$
\operatorname{Cov}(\boldsymbol{L} \boldsymbol{Y}) \geq \operatorname{Cov}\left(\boldsymbol{L}_{\Omega} \boldsymbol{Y}\right)=\operatorname{Cov}\left(\boldsymbol{U}_{\Omega} \boldsymbol{Y}\right)=\operatorname{Cov}(\widetilde{\boldsymbol{\psi}}),
$$

and the proof is complete.

We now look for an expression to $\widetilde{\psi}$ which exhibits the algebraic structure of models with COBS. Let the $g_{j}$ row vectors of $A_{j}$ constitute an orthonormal basis for $R\left(Q_{j}\right)$, so that we have

$$
\boldsymbol{A}_{j} \boldsymbol{A}_{j}^{\top}=\boldsymbol{I}_{g_{j}}, \quad \boldsymbol{A}_{j}^{\top} \boldsymbol{A}_{j}=\boldsymbol{Q}_{j}, j=1, \ldots, m
$$

we put $\boldsymbol{X}_{0, j}=\boldsymbol{A}_{j} \boldsymbol{X}_{j}$ and represent by $\boldsymbol{P}_{j}$ the orthogonal projection matrix on $\boldsymbol{\Omega}_{j}=R\left(\boldsymbol{X}_{0, j}\right), j=1, \ldots, m$. If, with $p_{j}=\operatorname{rank}\left(\boldsymbol{P}_{j}\right)$, the $p_{j}$ row vectors of $\boldsymbol{W}_{j}$ constitute an orthonormal basis for $\Omega_{j}$, we will have

$$
\boldsymbol{W}_{j} \boldsymbol{W}_{j}^{\top}=\boldsymbol{I}_{p_{j}}, \quad \boldsymbol{W}_{j}^{\top} \boldsymbol{W}_{j}=\boldsymbol{P}_{j}, j=1, \ldots, m .
$$

When $p_{j}=0$ we assume that $\boldsymbol{I}_{0}=[0]$ and that $\boldsymbol{P}_{j}=\mathbf{0}_{n \times n}$. 
We now establish

Proposition 3 In models with COBS, the $\dot{\boldsymbol{Q}}_{j}=\boldsymbol{Q}_{j} \boldsymbol{T}$ and the $\ddot{\boldsymbol{Q}}_{j}=\boldsymbol{A}_{j}^{\top} \boldsymbol{P}_{j} \boldsymbol{A}_{j}$ are identical orthogonal projection matrices with rank $p_{j}, j=1, \ldots, m$.

Proof If $p_{j}=0$ we have $\dot{\boldsymbol{Q}}_{j}=\ddot{\boldsymbol{Q}}_{j}=\mathbf{0}_{n \times n}$. We saw that in models with COBS the $\dot{\boldsymbol{Q}}_{j}, j=1, \ldots, m$ are orthogonal projection matrices it being straightforward to show that the $\ddot{\boldsymbol{Q}}_{j}, j=1, \ldots, m$, also are. Moreover

$R\left(\dot{\boldsymbol{Q}}_{j}\right)=R\left(\boldsymbol{Q}_{j} \boldsymbol{T}\right)=\boldsymbol{Q}_{j} R(\boldsymbol{T})=\boldsymbol{Q}_{j} R\left(\boldsymbol{X}_{0}\right)=\boldsymbol{A}_{j}^{\top} \boldsymbol{A}_{j} R\left(\boldsymbol{X}_{0}\right)=$ $\boldsymbol{A}_{j}^{\top} R\left(\boldsymbol{A}_{j} \boldsymbol{X}_{0}\right)=\boldsymbol{A}_{j}^{\top} R\left(\boldsymbol{X}_{0, j}\right)=\boldsymbol{A}_{j}^{\top} R\left(\boldsymbol{P}_{j}\right)=R\left(\boldsymbol{A}_{j}^{\top} \boldsymbol{P}_{j}\right)=R\left(\left(\boldsymbol{A}_{j}^{\top} \boldsymbol{P}_{j}\right)\left(\boldsymbol{A}_{j}^{\top} \boldsymbol{P}_{j}\right)^{\top}\right)$ $=R\left(\boldsymbol{A}_{j}^{\top} \boldsymbol{P}_{j} \boldsymbol{A}_{j}\right)=R\left(\ddot{\boldsymbol{Q}}_{j}\right)$, thus $\dot{\boldsymbol{Q}}_{j}=\ddot{\boldsymbol{Q}}_{j}$ and $\operatorname{rank}\left(\ddot{\boldsymbol{Q}}_{j}\right)=\operatorname{rank}\left(\dot{\boldsymbol{Q}}_{j}\right)$. Now $\boldsymbol{P}_{j}=\boldsymbol{A}_{j} \ddot{\boldsymbol{Q}}_{j} \boldsymbol{A}_{j}^{\top}$, so $p_{j}=\operatorname{rank}\left(\boldsymbol{P}_{j}\right)=\operatorname{rank}\left(\ddot{\boldsymbol{Q}}_{j}\right)=\operatorname{rank}\left(\dot{\boldsymbol{Q}}_{j}\right)$ and the proof is complete.

Corollary 2 In models with COBS and matrix $\boldsymbol{X}_{0}$ with $k$ linearly independent column vectors we have $k=\sum_{j=1}^{m} p_{j}$.

$\operatorname{Proof}$ We have $k=\operatorname{rank}\left(\boldsymbol{X}_{0}\right)=\operatorname{rank}(\boldsymbol{T})$ so the thesis follows from

$$
\boldsymbol{T}=\boldsymbol{I}_{n} \boldsymbol{T}=\left(\sum_{j=1}^{m} \boldsymbol{Q}_{j}\right) \boldsymbol{T}=\sum_{j=1}^{m} \dot{\boldsymbol{Q}}_{j}
$$

and from the $\dot{\boldsymbol{Q}}_{1}, \ldots, \dot{\boldsymbol{Q}}_{m}$ being pairwise orthogonal so that $\operatorname{rank}\left(\sum_{j=1}^{m} \dot{\boldsymbol{Q}}_{j}\right)=$ $\left(\sum_{j=1}^{m} \operatorname{rank}\left(\dot{\boldsymbol{Q}}_{j}\right)\right)=\sum_{j=1}^{m} p_{j}$.

Let us have $p_{j}>0$ if and only if $j \leq l$, with $l \leq m$, and put $\boldsymbol{Y}_{j}=\boldsymbol{A}_{j} \boldsymbol{Y}$ and $\boldsymbol{Z}_{j}=\boldsymbol{W}_{j} \boldsymbol{Y}_{j}, j=1, \ldots, l$. Since $\dot{\boldsymbol{Q}}_{j}=\mathbf{0}_{n \times n}$, if $j>l$, whenever $l<m$, we have

$$
\boldsymbol{T}=\sum_{j=1}^{l} \boldsymbol{Q}_{j} \boldsymbol{T}=\sum_{j=1}^{l} \dot{\boldsymbol{Q}}_{j}=\sum_{j=1}^{l} \ddot{\boldsymbol{Q}}_{j}=\sum_{j=1}^{l} \boldsymbol{A}_{j}^{\top} \boldsymbol{P}_{j} \boldsymbol{A}_{j}
$$

as well as, since $\boldsymbol{P}_{j}=\boldsymbol{W}_{j}^{\top} \boldsymbol{W}_{j}, j=1, \ldots, l$

$$
\begin{gathered}
\tilde{\boldsymbol{\mu}}=\boldsymbol{T} \boldsymbol{Y}=\sum_{j=1}^{l} \boldsymbol{A}_{j}^{\top} \boldsymbol{P}_{j} \boldsymbol{A}_{j} \boldsymbol{Y}=\sum_{j=1}^{l} \boldsymbol{A}_{j}^{\top} \boldsymbol{P}_{j} \boldsymbol{Y}_{j}= \\
=\sum_{j=1}^{l} \boldsymbol{A}_{j}^{\top} \boldsymbol{W}_{j}^{\top} \boldsymbol{W}_{j} \boldsymbol{Y}_{j}=\sum_{j=1}^{l} \boldsymbol{A}_{j}^{\top} \boldsymbol{W}_{j}^{\top} \boldsymbol{Z}_{j}
\end{gathered}
$$

so that $\tilde{\boldsymbol{\mu}}=\sum_{j=1}^{l} \boldsymbol{U}_{j} \boldsymbol{Z}_{j}$, with $\boldsymbol{U}_{j}=\boldsymbol{A}_{j}^{\top} \boldsymbol{W}_{j}^{\top}, j=1, \ldots, l$. 


\section{Model Characterization}

We now characterize models whose LSE are UBLUE. The estimable vectors of a model with mean vector $\boldsymbol{\mu}=\boldsymbol{X}_{0} \boldsymbol{\beta}_{o}$ are the

$$
\psi=\boldsymbol{U} \boldsymbol{\mu} .
$$

The corresponding linear unbiased estimators are the $\boldsymbol{\psi}^{*}=\boldsymbol{L} \boldsymbol{Y}$ with

$$
\boldsymbol{L} \in[\boldsymbol{\psi}]=\{\boldsymbol{L}: E(\boldsymbol{L} \boldsymbol{Y})=\boldsymbol{\psi}\},
$$

where $E($.$) indicates mean vector. We now establish$

Lemma 1 We have $E\left(\boldsymbol{L}_{1} \boldsymbol{Y}\right)=E\left(\boldsymbol{L}_{2} \boldsymbol{Y}\right)$ if and only if $\boldsymbol{L}_{1} \boldsymbol{T}=\boldsymbol{L}_{2} \boldsymbol{T}$.

Proof Since $E\left(\boldsymbol{L}_{l} \boldsymbol{Y}\right)=\boldsymbol{L}_{l} \boldsymbol{\mu}=\boldsymbol{L}_{l} \boldsymbol{T} \boldsymbol{\mu}, l=1,2$, the sufficient condition is established. Inversely, if $E\left(\boldsymbol{L}_{1} \boldsymbol{Y}\right)=E\left(\boldsymbol{L}_{2} \boldsymbol{Y}\right)$ we will have, whatever $\boldsymbol{\beta}_{0}$, $\boldsymbol{L}_{1} \boldsymbol{T} \boldsymbol{X}_{0} \boldsymbol{\beta}_{0}=\boldsymbol{L}_{2} \boldsymbol{T} \boldsymbol{X}_{0} \boldsymbol{\beta}_{0}$ so that $\boldsymbol{L}_{1} \boldsymbol{T} \boldsymbol{X}_{0}=\boldsymbol{L}_{2} \boldsymbol{T} \boldsymbol{X}_{0}$ and that $\left(\boldsymbol{L}_{1} \boldsymbol{T}-\boldsymbol{L}_{2} \boldsymbol{T}\right) \boldsymbol{X}_{0}=\mathbf{0}$, where $\mathbf{0}$ denotes a null matrix. Thus the row vectors of $\boldsymbol{W}=\boldsymbol{L}_{1} \boldsymbol{T}-\boldsymbol{L}_{2} \boldsymbol{T}=$ $\left(\boldsymbol{L}_{1}-\boldsymbol{L}_{2}\right) \boldsymbol{T}$ have to be orthogonal to $\boldsymbol{\Omega}=R\left(\boldsymbol{X}_{0}\right)$, but these vectors also belong to $\boldsymbol{\Omega}$ so they are null which gives $\boldsymbol{L}_{1} \boldsymbol{T}-\boldsymbol{L}_{2} \boldsymbol{T}=\mathbf{0}$ and so $\boldsymbol{L}_{1} \boldsymbol{T}=\boldsymbol{L}_{2} \boldsymbol{T}$ as we wanted to established.

Now the LSE for $\boldsymbol{\psi}=\boldsymbol{U} \boldsymbol{\mu}$ is

$$
\widetilde{\psi}=\boldsymbol{L}(\boldsymbol{\psi}) \boldsymbol{Y}
$$

with $\boldsymbol{L}(\psi)=\boldsymbol{U} \boldsymbol{T}$ and $\tilde{\boldsymbol{\mu}}=\boldsymbol{T} \boldsymbol{Y}$. We see that $\boldsymbol{L}(\boldsymbol{\psi}) \in[\boldsymbol{\psi}]$, since

$$
E(\widetilde{\boldsymbol{\psi}})=\boldsymbol{L}(\boldsymbol{\psi}) \boldsymbol{\mu}=\boldsymbol{U} \boldsymbol{T} \boldsymbol{X}_{0} \boldsymbol{\beta}_{0}=\boldsymbol{U} \boldsymbol{\mu}=\boldsymbol{\psi},
$$

besides this, according to Lemma $1, \boldsymbol{L} \in[\boldsymbol{\psi}]$ if and only if

$$
L T=L(\psi) T=U T T=U T=L(\psi) .
$$

Putting $\boldsymbol{T}^{c}=\boldsymbol{I}_{n}-\boldsymbol{T}$ we have, with $\boldsymbol{L} \in[\boldsymbol{\psi}]$,

$$
\boldsymbol{L}=\boldsymbol{L} \boldsymbol{T}+\boldsymbol{L} \boldsymbol{T}^{c}=\boldsymbol{L}(\boldsymbol{\psi})+r \boldsymbol{B},
$$

with $-\infty<r<+\infty$ and $\boldsymbol{B}=\frac{1}{r} \boldsymbol{L} \boldsymbol{T}^{c}$. Thus,

$$
\operatorname{Cov}_{\theta}(\boldsymbol{L Y})=\operatorname{Cov}_{\theta}(\boldsymbol{L}(\psi) \boldsymbol{Y})+2 r \operatorname{Cov}_{\theta}(\boldsymbol{L}(\psi) \boldsymbol{Y}, \boldsymbol{B} \boldsymbol{Y})+r^{2} \operatorname{Cov}_{\theta}(\boldsymbol{B} \boldsymbol{Y})
$$

it being easy to see that we have, whatever $r \in]-\infty ;+\infty[$,

$$
\operatorname{Cov}_{\theta}(\widetilde{\boldsymbol{\psi}})=\operatorname{Cov}_{\theta}(\boldsymbol{L}(\psi) \boldsymbol{Y}) \leq \operatorname{Cov}_{\theta}(\boldsymbol{L Y})
$$


if and only if $\operatorname{Cov}_{\theta}(\boldsymbol{L}(\psi) \boldsymbol{Y}, \boldsymbol{B Y})=\mathbf{0}$. Since $\boldsymbol{B}=\frac{1}{r} \boldsymbol{L} T^{c}$ we get

$$
\operatorname{Cov}_{\theta}\left(\boldsymbol{L T} \boldsymbol{Y}, \boldsymbol{L} \boldsymbol{T}^{c} \boldsymbol{Y}\right)=\mathbf{0},
$$

whenever

$$
\operatorname{Cov}_{\theta}(\boldsymbol{L}(\psi) \boldsymbol{Y}, \boldsymbol{B} \boldsymbol{Y})=\mathbf{0} .
$$

Now

$$
\begin{gathered}
\operatorname{Cov}_{\theta}\left(\boldsymbol{L} \boldsymbol{T} \boldsymbol{Y}, \boldsymbol{L} \boldsymbol{T}^{c} \boldsymbol{Y}\right)=\boldsymbol{L} \boldsymbol{T} \boldsymbol{V}(\boldsymbol{\theta}) \boldsymbol{T}^{c} \boldsymbol{L}^{\top}= \\
\left.\boldsymbol{L}\left[\boldsymbol{T} \boldsymbol{V}(\boldsymbol{\theta})\left(\boldsymbol{I}_{n}-\boldsymbol{T}\right)\right] \boldsymbol{L}^{\top}=\boldsymbol{L}[\boldsymbol{T} \boldsymbol{V}(\boldsymbol{\theta})-\boldsymbol{T} \boldsymbol{V}(\boldsymbol{\theta}) \boldsymbol{T})\right] \boldsymbol{L}^{\top},
\end{gathered}
$$

so that to have

$$
\operatorname{Cov}_{\theta}(\widetilde{\boldsymbol{\psi}}) \leq \operatorname{Cov}_{\theta}(\boldsymbol{L} \boldsymbol{Y})
$$

for every $\boldsymbol{\theta}$, if and only if $\boldsymbol{T} \boldsymbol{V}(\boldsymbol{\theta})-\boldsymbol{T} V(\boldsymbol{\theta}) \boldsymbol{T}=\boldsymbol{T} V(\boldsymbol{\theta}) \boldsymbol{T}^{c}=\mathbf{0}$, which gives $\boldsymbol{T} \boldsymbol{V}(\boldsymbol{\theta})=\boldsymbol{T} \boldsymbol{V}(\boldsymbol{\theta}) T$ and

$$
\boldsymbol{V}(\boldsymbol{\theta}) \boldsymbol{T}=(\boldsymbol{T} \boldsymbol{V}(\boldsymbol{\theta}))^{\top}=(\boldsymbol{T} \boldsymbol{V}(\boldsymbol{\theta}) \boldsymbol{T})^{\top}=\boldsymbol{T} \boldsymbol{V}(\boldsymbol{\theta}) \boldsymbol{T}=\boldsymbol{T} \boldsymbol{V}(\boldsymbol{\theta}),
$$

also for every $\boldsymbol{\theta}$.

We now establish

Theorem 2 The LSE are UBLUE if and only if, for every $\boldsymbol{\theta}, \boldsymbol{T}$ commutes with $\boldsymbol{V}(\boldsymbol{\theta})$, Proof The preceding discussion establishes the necessary condition. To complete the proof we point out that, when $\boldsymbol{T}$ commutes with $\boldsymbol{V}(\boldsymbol{\theta})$ we have

$$
\operatorname{Cov}_{\theta}(\boldsymbol{L} \boldsymbol{T} \boldsymbol{Y}, \boldsymbol{B} \boldsymbol{Y})=r \operatorname{Cov}_{\theta}\left(\boldsymbol{L} \boldsymbol{T} \boldsymbol{Y}, \frac{1}{r} \boldsymbol{L} \boldsymbol{T}^{c} \boldsymbol{Y}\right)=\boldsymbol{L} \boldsymbol{T} \boldsymbol{V}(\boldsymbol{\theta}) \boldsymbol{T}^{c} \boldsymbol{L}^{\top}=\mathbf{0}_{n \times n},
$$

and so

$$
\operatorname{Cov}_{\theta}(\boldsymbol{L} \boldsymbol{Y})=\operatorname{Cov}_{\theta}(\boldsymbol{L}(\boldsymbol{\psi}) \boldsymbol{Y})+r^{2} \operatorname{Cov}_{\theta}(\boldsymbol{B} \boldsymbol{Y}) \geq \operatorname{Cov}_{\theta} \boldsymbol{L}(\boldsymbol{\psi})=\operatorname{Cov}_{\theta}(\tilde{\boldsymbol{\psi}}) .
$$

Now the models with OBS where $\boldsymbol{T}$ commutes with the $\boldsymbol{M}_{1}, \ldots, \boldsymbol{M}_{w}$ and so with $\boldsymbol{V}(\boldsymbol{\theta})$, whatever $\boldsymbol{\theta}$, are those with COBS so these are the models with OBS whose LSE are UBLUE.

Corollary 3 Models with OBS have LSE that are UBLUE if and only if they have COBS. 
In establishing Theorem 2, we did not require that

$$
\boldsymbol{V}(\boldsymbol{\theta})=\sum_{i=1}^{w} \theta_{i} \boldsymbol{M}_{i}
$$

in order to widen the class of models to which our results applies. Moreover, as we stated in the introduction, when we restrict ourselves to OBS, assuming that

$$
\boldsymbol{V}(\boldsymbol{\gamma})=\sum_{j=1}^{m} \gamma_{j} \boldsymbol{Q}_{j}
$$

with $\boldsymbol{\gamma} \in \nabla_{\geq}$, our result holds whatever the dimension $(\leq m)$ of $\nabla$.

\section{Final Remarks}

The models we considered have variance-covariance matrices $\boldsymbol{V}(\boldsymbol{\gamma})=\sum_{j=1}^{m} \gamma_{j} \boldsymbol{Q}_{j}$ where the $\boldsymbol{Q}_{1}, \ldots, \boldsymbol{Q}_{m}$ are POOPM that add up to $\boldsymbol{I}_{n}$, and $\boldsymbol{\gamma} \in \nabla$ with $\operatorname{dim}(\nabla)=$ $r \geq 1$. We discussed the role played by $\boldsymbol{T}$, the orthogonal projection matrix on the space spanned by the mean vector, commuting with the $\boldsymbol{Q}_{1}, \ldots, \boldsymbol{Q}_{m}$ in the LSE of estimable vectors being UBLUE, this is, being BLUE whatever $\boldsymbol{\gamma}$. Namely we showed that commutativity characterizes the models, in the class we consider, whose LSE are UBLUE. We point out that in our mixed models we had $\gamma \in R\left(\boldsymbol{B}^{\top}\right)_{\geq}$. To have, as required in $[8,9]$, the $\boldsymbol{\gamma} \in \mathbb{R}_{\geq}^{m}$, matrix $\boldsymbol{B}$ would have to have rank $m$ and thus being invertible. This condition holds when $\boldsymbol{M}$ is a basis for $\boldsymbol{M}$, we then say, see [4], that the family $\boldsymbol{M}$ is perfect.

Acknowledgements This work was partially supported by national funds of FCT-Foundation for Science and Technology under UID/MAT/00212/2013 and UID/MAT/00297/ 2013.

\section{References}

1. Bailey, R. A., Ferreira, S. S., Ferreira, D., \& Nunes, C. (2016). Estimability of variance components when all model matrices commute. Linear Algebra and its Applications, 492, 144-160.

2. Caliński, T., \& Kageyama, S. (2000). Block designs: a randomization approach (Vol. I) Analysis. Lecture Notes in Statistics, 150. New York: Springer-Verlag.

3. Caliński, T., \& Kageyama, S. (2003). Block designs: a randomization approach (Vol. II) Design. Lecture Notes in Statistics, 170. New York: Springer-Verlag.

4. Ferreira, S. S., Ferreira, D., Fernandes, C., \& Mexia, J. T. (2007). Orthogonal Mixed Models and Perfect Families of Symmetric Matrices. 56th Session of the International Statistical Institute, Book of Abstracts, ISI, Lisboa, 22 a 29 de Agosto. 
5. Fonseca, M., Mexia, J. T., \& Zmyślony, R. (2006). Binary operations on Jordan algebras and orthogonal normal models. Linear Algebra and its Applications, 417, 75-86.

6. Fonseca, M., Mexia, J. T., \& Zmyslony, R. (2008). Inference in normal models with commutative orthogonal block structure. Acta et Commentationes Universitatis Tartunesis de Mathematica, 12, 3-16.

7. Mexia, J. T., Vaquinhas, R., Fonseca, M., \& Zmyślony, R. (2010). COBS: segregation, matching, crossing and nesting. Latest trends on applied mathematics, simulation, modeling. In 4th International Conference on Applied Mathematics, Simulation, Modelling (ASM'10) (pp. 249-255).

8. Nelder, J. A. (1965). The analysis of randomized experiments with orthogonal block structure. I. Block structure and the null analysis of variance. Proceedings of the Royal Society of London, Series A, 273, 147-162.

9. Nelder, J. A. (1965). The analysis of randomized experiments with orthogonal block structure. II. Treatment structure and the general analysis of variance. Proceedings of the Royal Society of London, Series A, 273, 163-178.

10. Seely, J. (1971). Quadratic subspaces and completeness. Annals of Mathematical Statistics, 42, 710-721.

11. VanLeeuwen, D. M., Birkes, D. S., \& Seely, J. F. (1999). Balance and orthogonality in designs for mixed classification models. Annals of Statistics, 27(6), 1927-1947.

12. VanLeeuwen, D. M., Seely, J. F., \& Birkes, D. S. (1998). Sufficient conditions for orthogonal designs in mixed linear models. Journal of Statistical Planning and Inference, 73, 373-389.

13. Zmyślony, R. (1980). A characterization of best linear unbiased estimators in the general linear model. In Mathematical Statistics and Probability Theory, Proceedings of the Sixth International Conference, Wisla 1978, Lecture Notes in Statistics, 2 (pp. 365-373). Berlin: Springer.

14. Zyskind, G. (1967). On canonical forms, non-negative covariance matrices and best simple linear least squares estimators in linear models. Annals of Mathematical Statistics, 38, 10921109. 